EPJ Web of Conferences 41, 11003 (2013)

DOI: $10.1051 /$ epjconf/20134111003

C) Owned by the authors, published by EDP Sciences, 2013

\title{
Generation of phase-stable half-cycle mid-infrared pulses through filamentation in gases
}

\author{
Yutaka Nomura $^{1}$, Takao Fuji ${ }^{1, a}$, Hideto Shirai ${ }^{2}$, Noriaki Tsurumachi ${ }^{2}$, Alexander A. Voronin ${ }^{3}$, and \\ Aleksei M. Zheltikov ${ }^{3,4}$ \\ 1 Institute for Molecular Science, 38 Nishigonaka, Myodaiji, Okazaki 444-8585, Japan \\ 2 Faculty of Engineering, Kagawa University, 2217-20 Hayashi-cho, Takamatsu, 761-0396, Japan \\ 3 Physics Department, International Laser Center, M.V. Lomonosov Moscow State University, 119992 \\ Moscow, Russia \\ 4 Department of Physics and Astronomy, Texas A\&M University, College Station, Texas 77843-4242, \\ USA
}

\begin{abstract}
Phase-stable half-cycle mid-infrared pulses were produced through filamentation in argon. The pulse width was obtained as $7.9 \mathrm{fs}$ at $4.1 \mu \mathrm{m}$ carrier wavelength by using frequency-resolved optical gating.
\end{abstract}

Filamentation of powerful ultrashort laser pulses in gases [1-3] is one of the most interesting phenomena in nonlinear optics. The balance between self-focusing and plasma self-defocusing makes the pulse propagate much longer than the Rayleigh range with a very high intensity. It results in a dramatic enhancement of nonlinear processes occurring in the filamentation zone. This phenomenon enables high intensity pulse compression and efficient nonlinear wavelength conversion with gas media [2,3].

Enhanced nonlinear-optical processes in laser-induced filaments suggest a new strategy for the generation of ultrashort pulses of long-wavelength radiation. Ultrabroadband mid-infrared (MIR, 3$20 \mu \mathrm{m})$ pulse generation through filamentation in air was firstly demonstrated in 2007 [4], and the technique was followed by several groups [5-7]. Such MIR pulses with more than one octave at full width at half maximum are very attractive to be applied for molecular spectroscopy, e.g. two-dimensional infrared spectroscopy.

In this contribution, we report the latest progress of the ultrabroadband MIR pulse generation through filamentation. By using argon gas as the nonlinear medium and purging the optical path for the MIR pulse with the argon gas, a long tail of the pulse due to free induction decay of atmospheric carbon dioxide and water vapor disappeared, and it was possible to achieve generation of high contrast MIR pulses. Full characterization of the pulse shape of the MIR field indicates that its pulse duration was $7.9 \mathrm{fs}$, which is about half-cycle period of the center wavelength $(4.1 \mu \mathrm{m})$ of the pulse.

The experimental setup is shown in Fig. 1. The light source was based on a Ti:sapphire multi-pass amplifier system (Femtolasers, $800 \mathrm{~nm}, 25 \mathrm{fs}, 0.9 \mathrm{~mJ}$ at $1 \mathrm{kHz})$. The second harmonic $\left(\omega_{2}, 25 \mu \mathrm{J}\right)$ and fundamental $\left(\omega_{1}, 675 \mu \mathrm{J}\right)$ pulses were spatially and temporally overlapped and focused into argon by a concave mirror $(r=-1000 \mathrm{~mm})$, generating a bright filament with a length of $\sim 3 \mathrm{~cm}$ around the beam focus. This filament generated an MIR pulse $\left(\omega_{0}\right)$ through an ionization-assisted wave mixing process $\left(\omega_{1}+\omega_{1}-\omega_{2} \rightarrow \omega_{0}\right)$. The energy of this MIR pulse was measured as $\sim 250 \mathrm{~nJ}$ by using a pyroelectric detector (J-10MB-LE, Coherent). With this energy level, it is possible to apply the pulses for the nonlinear spectroscopy of condensed matter. The pulse-to-pulse intensity fluctuation was about $2.5 \% \mathrm{rms}$

\footnotetext{
a e-mail: fuji@ims.ac.jp
}

This is an Open Access article distributed under the terms of the Creative Commons Attribution License 2.0, which permits unrestricted use, distribution, and reproduction in any medium, provided the original work is properly cited. 
In order to quantitatively evaluate the temporal shape of the generated MIR pulse, we measured cross-correlation frequency-resolved optical gating (XFROG)[8]. We used argon again as a nonlinear medium and used four-wave mixing process $\left(\omega_{1}+\omega_{1}-\omega_{0} \rightarrow \omega_{2}\right)$ as a nonlinear interaction between the test pulse (MIR pulse, $\left.E_{\text {test }}(t)\right)$ and the reference pulse $\left(E_{\text {ref }}(t)\right)$. The scheme is free from spectral filtering caused by phase matching condition in the nonlinear interaction.

The system for the XFROG measurement is also shown in Fig. 1. Small portion $(\sim 2 \mu \mathrm{J})$ of the fundamental 25-fs pulse was used as a reference pulse. The reference pulse and the MIR pulse (test pulse) were combined through a mirror with a hole and focused into argon with an aluminium-coated parabolic mirror $(f=150 \mathrm{~mm})$. Generated blue spectra (centered around $440 \mathrm{~nm}$ ) were measured with a spectrometer (USB2000+, OceanOptics) by scanning the delay time ( $\tau$ in Fig. 1 ) between the reference pulse and the MIR test pulse. The reference pulse was independently characterized with SHG-FROG, and the result was used for retrieving the MIR pulse.

The XFROG signal $I_{\mathrm{FROG}}(\nu, \tau)$ is written as follows,

$$
\begin{aligned}
E_{\mathrm{sig}}(t, \tau) & \propto E_{\mathrm{ref}}(t)^{2} E_{\mathrm{test}}^{*}(t-\tau), \\
I_{\mathrm{FROG}}(v, \tau) & =\left|\int d t E_{\mathrm{sig}}(t, \tau) \exp (i 2 \pi v t)\right|^{2},
\end{aligned}
$$

where the electric fields of the reference pulse, the test pulse, and the four-wave mixing signal are $E_{\text {ref }}(t), E_{\text {test }}(t)$, and $E_{\text {sig }}(t, \tau)$, respectively.

The measured and the retrieved XFROG traces are shown in Fig. 2(a) and (b). The main feature of the trace indicates that the residual chirp of the test pulse is very small. The FROG error was 0.003 with $256 \times 256$ grid. The retrieved time and frequency domain pictures are shown in Fig. 2(c) and (d), respectively. The pulse width is estimated to be $7.9 \mathrm{fs}$, which is 0.58 cycles for $4.1 \mu \mathrm{m}$ carrier wavelength. The retrieved spectrum is spread over whole MIR region $\left(500-5000 \mathrm{~cm}^{-1}\right)$. The broadness of the spectrum was due to the weak dispersion of the medium, with the phase-matching length exceeding the length of the filament for all the MIR spectral components observed in the experiments.

In theory, the carrier-envelope phase (CEP) of the generated MIR pulse is passively stabilized with the present scheme [4,9]. Following the expression of CEP in frequency conversion processes described in Ref. [9], the phase of the generated MIR pulse is given by $\Psi_{\omega 0}=\pi / 2+\Psi_{\omega 1}+\Psi_{\omega 1}-\Psi_{\omega 2}$, where $\Psi_{\omega 0}, \Psi_{\omega 1}$, and $\Psi_{\omega 2}$ are the phases of $\omega_{0}, \omega_{1}$, and $\omega_{2}$ components, respectively. Since $\Psi_{\omega 2}=$ $\pi / 2+2 \Psi_{\omega 1}, \Psi_{\omega 0}$ must be 0 , which fact means that the CEP of the MIR pulse is automatically stabilized even without CEP stabilization of the input pulse. This feature makes the scheme highly attractive since CEP is very important physical property of sub-single cycle pulses. It is noted that the delay line between the fundamental and the second harmonic pulse ( $T$ in Fig. 1$)$ must be stabilized in wavelength

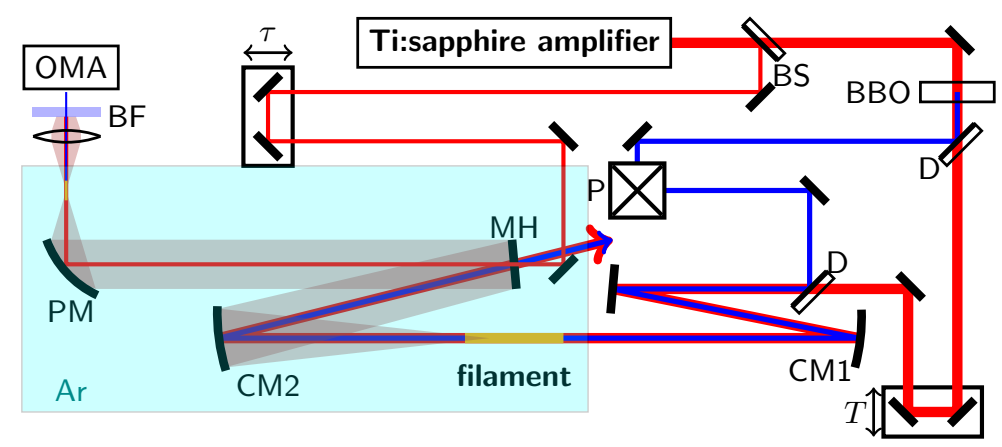

Fig. 1. Schematic of the system. The shaded area was purged with argon. BS: beam splitter (5\% reflection), BBO: $\beta$ - $\mathrm{BaB}_{2} \mathrm{O}_{4}$ crystal (Type $1, \theta=29^{\circ}, t=0.1 \mathrm{~mm}$ ), D: dichroic mirror, P: periscope, CM1: $r=1$ m concave mirror, CM2: $r=0.5$ m concave mirror, MH: aluminium-coated mirror with a hole $(\phi=7 \mathrm{~mm})$, PM: aluminium-coated parabolic mirror, BF: bandpass filter for 335-610 nm (FGB37, Thorlabs), OMA: spectrometer for ultraviolet region (USB2000+, OceanOptics). 

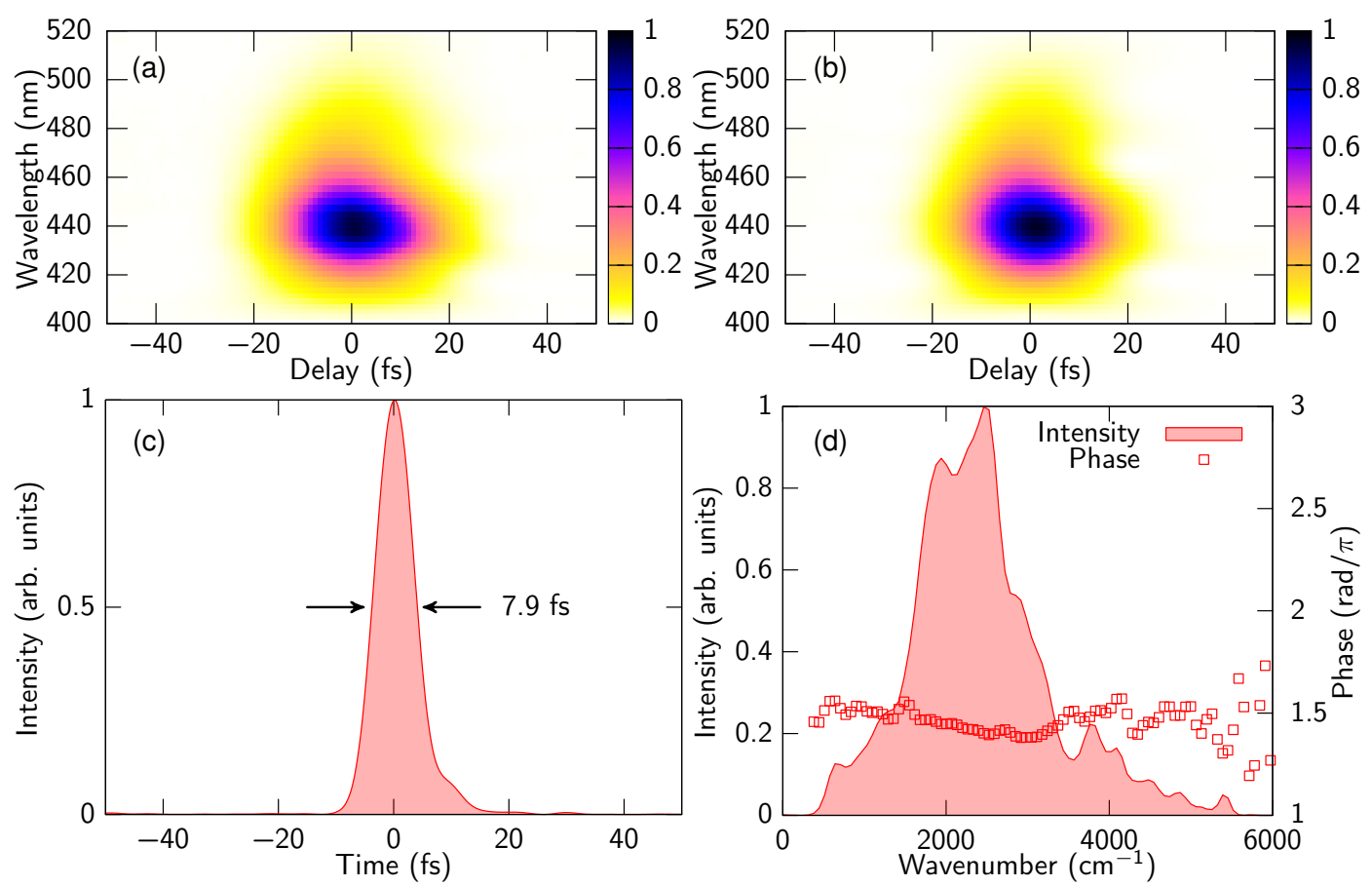

Fig. 2. (a) Experimental and (b) retrieved XFROG traces. The retrieved pulse in (c) time and (d) frequency domain.

accuracy[10,11]. This, however, is considerably easier than stabilizing the CEP phase of the input laser pulse itself.

In conclusion, ultrabroadband coherent MIR spectrum which covers the entire MIR region was generated through two-color filamentation. Due to the spatial and temporal quality, ultrashort MIR pulses generated in two-color filaments are ideal for numerous applications. In our experiments, MIR pulses as short as $7.9 \mathrm{fs}$ were generated, which corresponds to about a half-cycle of $4.1 \mu \mathrm{m}$ center wavelength.

\section{References}

1. A. Braun, G. Korn, X. Liu, D. Du, J. Squier, G. Mourou, Opt. Lett. 20, 73 (1995)

2. L. Bergé, S. Skupin, R. Nuter, J. Kasparian, J.P. Wolf, Rep. Prog. Phys. 70, 1633 (2007)

3. A. Couairon, A. Mysyrowicz, Phys. Rep. 441, 47 (2007)

4. T. Fuji, T. Suzuki, Opt. Lett. 32, 3330 (2007)

5. F. Théberge, M. Châteauneuf, G. Roy, P. Mathieu, J. Dubois, Phys. Rev. A 81, 033821 (2010)

6. P.B. Petersen, A. Tokmakoff, Opt. Lett. 35, 1962 (2010)

7. M.D. Thomson, V. Blank, H.G. Roskos, Opt. Express 18, 23173 (2010)

8. S. Linden, H. Giessen, J. Kuhl, Phys. Status Solidi B 206, 119 (1998)

9. A. Baltuška, T. Fuji, T. Kobayashi, Phys. Rev. Lett. 88, 133901 (2002)

10. T. Fuji, N. Ishii, C.Y. Teisset, X. Gu, T. Metzger, A. Baltuška, N. Forget, D. Kaplan, A. Galvanauskas, F. Krausz, Opt. Lett. 31, 1103 (2006)

11. X. Xie, J. Dai, X.C. Zhang, Phys. Rev. Lett. 96, 075005 (2006) 